\title{
Commentary: Neural correlates of expected risks and returns in risky choice across development
}

\author{
Faisal Mushtaq ${ }^{1}$, Liam J. B. Hill ${ }^{1}$, Amy R. Bland ${ }^{2}$, Matt Craddock ${ }^{1}$ and Neil B. Boyle ${ }^{1 *}$ \\ ${ }^{1}$ School of Psychology, Faculty of Medicine and Health, University of Leeds, Leeds, UK, ${ }^{2}$ Neuroscience and Psychiatry Unit, \\ University of Manchester, Manchester, UK
}

Keywords: decision making, risk-taking, development, fMRI, neuroscience of decision making, cognitive development, neuroeconomics

\section{A Commentary on}

Neural correlates of expected risks and returns in risky choice across development by van Duijvenvoorde, A. C. K., Huizenga, H. M., Somerville, L. H., Delgado, M. R., Powers, A., Weeda, W. D., et al. (2015) J. Neurosci. 35, 1549-1560. doi: 10.1523/JNEUROSCI.1924-14.2015

"Wisdom comes with age" is an oft-heard expression. It suggests that across development we
improve in our ability to make decisions-but evidence for its validity is equivocal. In real-
world decision-making, there is an adolescent-specific increased propensity to engage in behaviors
associated with morbidity and mortality (e.g., road traffic accidents, unprotected sex, violence, drug,
and alcohol abuse; Blum and Nelson-Mmari, 2004). However, this inverted u-shape developmental
trajectory for risk-taking is typically not observed in laboratory-based studies (Defoe et al., 2015).
As such, there exists a need to: (a) bridge the gap between laboratory and real world behavior;
and (b) clarify the processes underlying developmental differences in decision-making to inform
interventions that target a reduction in health-risking adolescent activities.

OPEN ACCESS

Edited by:

Shuhei Yamaguchi,

Shimane University, Japan

Reviewed by:

Bernd Figner,

Columbia University, USA

*Correspondence:

Neil B. Boyle,

n.b.boyle@leeds.ac.uk

Received: 22 April 2015 Accepted: 18 June 2015 Published: 30 June 2015

Citation:

Mushtaq F, Hill LJB, Bland AR, CraddockM and Boyle NB (2015) Commentary: Neural correlates of expected risks and returns in risky choice across development.

Front. Hum. Neurosci. 9:388. doi: 10.3389/fnhum.2015.00388
A recent fMRI study by Van Duijvenvoorde et al. (2015) examined the neural correlates of risk-taking in three maturational periods: childhood, adolescence, and adulthood. Participants from each age group performed a version of the Columbia Card Task (CCT; Figner et al., 2009). In the fMRI-CCT participants are shown up to 16 cards face down per round-each card is associated with a gain or loss of reward and participants are informed about the proportion of cards associated with loss and gain during each round. Participants turn over cards until they either decide to stop or until they turn over a loss card, which ends the round. Loss probability increases with each card selection - as each gain card drawn reduces the proportion of gain cards in the remaining set. This follows a "Risk-Return" approach to understanding decision-making. In economics these tasks are used to isolate risky decisions into two components: (i) a returnwhich provides a measure of the expected value of the choice; and (ii) a risk component-which provides an index of outcome variability (e.g., SD of possible outcomes; Mushtaq et al., 2013). The degree to which a participant balances the potential reward for turning over another card with the potential risk allows a clear characterization of an individual's risk-taking behavior (Weber, 2010). This model, novel to developmental neuroimaging studies, has a clear advantage over classic utility-based models (e.g., tasks centered around prospect theory), which have difficulty in parsing out the contributions of risk and reward in decision-making. This is crucial for linking neural data to underlying cognitive processes as it allows researchers to tease out differences in risk and return sensitivity along the developmental pathway. Indeed, many previous imaging studies on developmental differences in the neural substrates of risk-taking confound these components (see Richards et al., 2013 review)-leading to an impasse in our understanding of how risk processing 
alone might vary over development. van Duijvenvoorde et al.'s study directly tackles this issue.

The ultimate goal of neuroimaging is to relate neural processes to behavior, but frequently fMRI analysis is limited to the production of statements regarding the relative magnitude of distinct conditions. Van Duijvenvoorde et al. (2015) study is an example of the value that can be obtained from mapping the relationship between brain and behavior. The decomposition of task performance into risk- and return-sensitivity allowed the identification of distinct areas that parametrically coded risk and return. Another interesting take on data analysis was the use of linear and quadratic models. The authors reasoned that if developmental changes followed a monotonic pattern over time, a linear model could describe them. If the effects were asymmetrically sensitive to adolescence specific effects, a quadratic function would best fit the data. These innovative approaches, through task employed and analysis procedure, allowed the authors to elucidate the specific neural mechanisms underlying the full dynamic of risky decision-making across differential maturational stages.

The results show the independence of reward and risk related cognitive processes in neural circuitry and patterns of maturation. Specifically, the researchers observed heightened activation for risk in adolescents in regions previously associated with risk processing, namely the right anterior insula, inferior frontal gyrus and the dorsal medial PFC (consistent with Preuschoff et al., 2008; Mohr et al., 2010). Importantly, adolescents' activations in these regions significantly correlated with their risk-sensitivity. In contrast, return related activity increased linearly with age in ventral medial PFC and posterior cingulate cortex (PPC) and correlated positively with behavioral return sensitivity. This approach provides two critical insights into developmental differences in decision-making hitherto unknown and would be difficult to parse out with traditional

\section{References}

Blum, R. W., and Nelson-Mmari, K. (2004). The health of young people in a global context. J. Adolesc. Health 35, 402-418. doi: 10.1016/S1054-139X(03)0 0537-8

Defoe, I. N., Dubas, J. S., Figner, B., and van Aken, M. A. G. (2015). A meta-analysis on age differences in risky decision making: adolescents versus children and adults. Psychol. Bull. 141, 48-84. doi: 10.1037/a0038088

Figner, B., Mackinlay, R. J., Wilkening, F., and Weber, E. U. (2009). Affective and deliberative processes in risky choice: age differences in risk taking in the Columbia Card Task. J. Exp. Psychol. Learn. Mem. Cogn. 35, 709-730. doi: 10.1037/a0014983

Gilaie-Dotan, S., Tymula, A., Cooper, N., Kable, J. W., Glimcher, P. W., and Levy, I. (2014). Neuroanatomy predicts individual risk attitudes. J. Neurosci. 34, 12394-12401. doi: 10.1523/JNEUROSCI.1600-14.2014

Mohr, P. N. C., Biele, G., and Heekeren, H. R. (2010). Neural processing of risk. J. Neurosci. 30, 6613-6619. doi: 10.1523/JNEUROSCI.0003-1 0.2010

Mushtaq, F., Stoet, G., Bland, A., and Schaefer, A. (2013). Relative changes from prior reward contingencies can constrain brain correlates of outcome monitoring. PLoS ONE 8:e66350. doi: 10.1371/journal.pone.0066350

Preuschoff, K., Quartz, S. R., and Bossaerts, P. (2008). Human insula activation reflects risk prediction errors as well as risk. J. Neurosci. 28, 2745-2752. doi: 10.1523/JNEUROSCI.4286-07.2008 decision-making tasks: (1) they show qualitatively different developmental changes in processing of risk and reward; and (2) fMRI results demonstrate a heightened adolescent specific response to the processing of risk.

These findings are an important first step and give rise to a number of interesting questions for future research. For example, in van Duijvenvoorde et al.'s study the individual variability in risk propensity was amplified in the adolescent group. To account for similar inter-individual variability, decision scientists have increasingly begun to focus on individual risk-preference and traits as decision predictors- ideologically moving from a nomothetic to idiographic approach (Stanovich and West, 2000). In other words, there has been an increased focus on individual risk-preference and traits as decision predictors alongside, or instead of, aggregate group-level analysis. Relatedly, another recent study (Gilaie-Dotan et al., 2014) reported gray matter volume in the right PPC can predict an individual's risk attitude. This may well-serve as a neural biomarker for risk propensity. Whilst such results are not causal in nature, they may provide a means of accounting for some proportion of the differences in adolescence. Convergence between such structural and functional neural evidence offers considerable scope for future research.

To summarize, Van Duijvenvoorde et al.'s (2015) study provides novel insights into the neural mechanisms of reward and risk sensitivity across maturation. Importantly, it delineates the neural circuitry involved in the etiology of risk processing and in doing so uncovers age-related neural differences that, with further investigation, may help explain variability in riskpropensity across the lifespan. Given the financial and health impact of sub-optimal risk seeking, being able to identify associations between brain, development and behavior holds promise to improve risk-avoidance interventions, thus providing substantial economic and societal benefit.

Richards, J. M., Plate, R. C., and Ernst, M. (2013). A systematic review of fMRI reward paradigms used in studies of adolescents vs. adults: the impact of task design and implications for understanding neurodevelopment. Neurosci. Biobehav. Rev. 37, 976-991. doi: 10.1016/j.neubiorev.2013.03.004

Stanovich, K. E., and West, R. F. (2000). Individual differences in reasoning: implications for the rationality debate? Behav. Brain Sci. 23, 645-665. discussion: 665-726. doi: 10.1017/S0140525X00003435

Van Duijvenvoorde, A. C. K., Huizenga, H. M., Somerville, L. H., Delgado, M. R., Powers, A., Weeda, W. D., et al. (2015). Neural correlates of expected risks and returns in risky choice across development. J. Neurosci. 35, 1549-1560. doi: 10.1523/JNEUROSCI.1924-14.2015

Weber, E. U. (2010). Risk attitude and preference. Wiley Interdiscip. Rev. Cogn. Sci. 1, 79-88. doi: $10.1002 /$ wcs. 5

Conflict of Interest Statement: The authors declare that the research was conducted in the absence of any commercial or financial relationships that could be construed as a potential conflict of interest.

Copyright (C) 2015 Mushtaq, Hill, Bland, Craddock and Boyle. This is an open-access article distributed under the terms of the Creative Commons Attribution License (CC $B Y)$. The use, distribution or reproduction in other forums is permitted, provided the original author(s) or licensor are credited and that the original publication in this journal is cited, in accordance with accepted academic practice. No use, distribution or reproduction is permitted which does not comply with these terms. 